\title{
Visualizing the effect additives have on the nanostructure of individual bio-inspired calcite crystals
}

\author{
J. Ihli', Y.-Y. Kim², M. Levenstein ${ }^{2}$, Y. Ning ${ }^{3}$, K. Wakonig ${ }^{1}$, M. Holler ${ }^{1}$, S. Armes ${ }^{3}$, R. Harder ${ }^{4}$, I. Robinson ${ }^{5}$, \\ F. Meldrum ${ }^{2}$ \\ ${ }^{1}$ Paul Scherrer Institute, Villigen PSI, Switzerland, \\ ${ }^{2}$ University of Leeds, Leeds, United Kingdom, \\ ${ }^{3}$ University of Sheffield, Sheffield, United Kingdom, \\ ${ }^{4}$ Argonne National Laboratory, Lemont, United States, \\ ${ }^{5}$ University College London, London, United Kingdom \\ Johannes.Ihli@psi.ch
}

Additives provide a versatile strategy for controlling crystallization processes, enabling selection of properties including crystal sizes, morphologies, and structures. The additive species can also be incorporated within the crystal and even the crystal lattice itself, leading for example to enhanced mechanical properties. However, while many techniques are available for analysing particle shape and structure, it remains challenging to characterize the structural inhomogeneities and defects introduced into individual crystals by these additives, where these govern many important material properties. Here, we exploit coherent diffraction imaging methods to visualize the distribution of additives within as well as the effects additives have on the internal structure of individual calcite crystals. Highlighted are how factors including supersaturation, solution composition and additive-crystal interactions govern the distribution of additives in single crystals. Further, emphasized is the emergence of a range of complex strain and zonation patterns depending on the nature of the additive, diverging in part and locally from commonly suggested distribution models. This work contributes to our understanding of the factors that govern the structure-property relationships of crystalline materials, where a controlled utilization of additives will ultimately inform the design of next-generation materials.

Keywords: BCDI, PXCT, Calcite, Additives, Composite 